\title{
Chiral restoration effects on the shear viscosity of a pion gas
}

\author{
Klaus Heckmann, ${ }^{1}$ Michael Buballa, ${ }^{1}$ and Jochen Wambach ${ }^{1,2}$ \\ ${ }^{1}$ Institut für Kernphysik, Technische Universität Darmstadt, Germany \\ ${ }^{2}$ GSI Helmholtz-Zentrum für Schwerionenforschung, Darmstadt, Germany
}

(Dated: October 2012)

\begin{abstract}
We investigate the shear viscosity of a pion gas in relativistic kinetic theory, using the Nambu-JonaLasinio model to construct the pion mass and the $\pi \pi$-interaction at finite temperature. Whereas at low temperatures the scattering properties and, hence, the viscosity are in agreement with lowestorder chiral perturbation theory, we find strong medium modifications in the crossover region. Here the system is strongly coupled and the scattering lengths diverge, similarly as for ultra-cold Fermi gases at a Feshbach resonance. As a consequence, the ratio $\eta / s$ is found to be strongly reduced as compared to calculations without medium-modified masses and scattering amplitudes. However, the quantitative results are very sensitive to the details of the applied approximations.

PACS numbers: $24.85 .+\mathrm{p}, 25.75 .-\mathrm{q}, 24.10 . \mathrm{Cn}, 24.10 . \mathrm{Jv}$
\end{abstract}

\section{INTRODUCTION}

The elliptic flow at RHIC was found to be in rather good agreement with almost ideal hydrodynamic behavior $[1$ [7]. This led to an intensive discussion of the hydrodynamical properties of strong-interaction (QCD) matter under extreme conditions. The viscous properties of a thermal system are characterized by its transport coefficients. These describe the deviation from ideal hydrodynamics and are determined by the micro-physics.

While originally the RHIC data have been interpreted as evidence for an almost perfect fluid in the quarkgluon phase, it was pointed out recently that the elliptic flow crucially depends on the transport properties in the hadronic phase [8]. In this phase, at low temperatures and small baryo-chemical potentials, the thermal properties of QCD matter are governed by the lightest degrees of freedom, namely pions. Moreover, for small enough temperatures, a gas of pions is sufficiently dilute to apply relativistic kinetic theory. Such a kinetic description of transport processes requires solely the (in-medium) masses and scattering amplitudes of the underlying particles. In this context, usually the vacuum values of these quantities are used [9 17]. The temperature dependence of the transport coefficients is then the result of the different thermal occupation of states. However, the pion mass and the pion interactions are modified by the hot and dense medium. Since pions are (near) Goldstonebosons of spontaneously broken chiral symmetry, their properties are sensitive to its restoration at high temperatures, especially in the chiral crossover region.

A consistent description of pion scattering processes in the vacuum and at low temperatures can be obtained within chiral perturbation theory [18 23]. However, the expansion breaks down in the chiral crossover region where nonperturbative effects become essential. Therefore, in order to describe $\pi \pi$-scattering in this region, an approach is needed which allows for a proper description of the chiral phase transition. The starting point for our investigations will be the Nambu-Jona-Lasinio (NJL) model [24, 25], which has been used extensively to study spontaneous chiral symmetry breaking in the vacuum and its restoration at finite temperature and (net) quark density. (For reviews see [26 29].) Unlike in the linear sigma model, which was employed in ref. [30] in a similar context, the elementary degrees of freedom in the NJL model are quarks. Mesons are constructed from $q \bar{q}-$ correlations. At low temperatures, the pion emerges as a bound state in the pseudoscalar channel, whose mass, decay constant and scattering properties are consistent with chiral low-energy theorems [31, 32]. However, in the vicinity of chiral restoration, the pion gets dissolved and becomes a broad resonance at high temperatures. This feature, which is a natural consequence of the compositeness, cannot be described properly in the linear sigma model and is our main motivation to use the NJL model.

In the present work, we focus on the shear viscosity $\eta$. This transport coefficient is essential for the behavior of the elliptic flow in non-central heavy-ion collisions. Moreover, the ratio of shear viscosity over entropy density, $\eta / s$, is a measure for the proximity of a given system to ideal fluid dynamical behavior. In the context of the AdS/CFT correspondence it has been conjectured that $\eta / s$ has a universal lower bound of $1 / 4 \pi$ [33] (although this con- 
jecture has been questioned by some authors, see, e.g., Ref. 34]). Thus, an almost-ideal fluid should acquire a value of $\eta / s$ which is close to this putative lower bound. Besides the hot and dense fireball of a heavy-ion collision, nearly-ideal hydrodynamic behavior has also been discussed for a system of ultra-cold fermionic atoms in a trap. Here a divergent scattering length (unitary limit) can be achieved via a Feshbach resonance tuned with an external magnetic field. Ultra-cold Fermi gases thus offer the exciting possibility to study strongly coupled systems under 'controlled conditions' in the laboratory and infer analogies to QCD matter. We shall see that a divergent scattering length can also be found in a pion gas near the chiral crossover.

This article is organized as follows. In sect. II we describe the main steps for calculating the shear viscosity in relativistic kinetic theory. In sect. III, we employ the NJL model to determine the relevant quantities which enter the kinetic description: After briefly reviewing the temperature dependence of quark and meson masses as well as the $\pi \pi$-scattering lengths, we introduce several approximation steps of increasing sophistication to calculate the in-medium $\pi \pi$-scattering amplitude and discuss the resulting changes of the total cross sections. The results for the shear viscosity and for $\eta / s$ are presented in sect. IV] Finally, in sect. V. we discuss the implications of our results and propose further developments.

\section{SHEAR VISCOSITY FROM KINETIC THEORY}

In this section we summarize the main ideas of how to calculate the shear viscosity within kinetic theory and list the corresponding equations that need to be solved. We largely follow Refs. [13, 15 17], and refer to these articles for further details.

The basic ingredients of relativistic hydrodynamics are the local fluid four-velocity $u^{\mu}(x)$ and the energymomentum tensor $T^{\mu \nu}$. Performing an expansion in gradients of $u$, the latter can be written as

$$
T^{\mu \nu}=T^{(0) \mu \nu}+T^{(1) \mu \nu}+\ldots
$$

where

$$
T^{(0) \mu \nu}=(\epsilon+p) u^{\mu} u^{\nu}-p g^{\mu \nu}
$$

is the ideal part solely specified by the local energy den- sity $\epsilon$ and pressure $p$, while

$$
\begin{aligned}
T^{(1) \mu \nu} & =\eta\left[\partial^{\mu} u^{\nu}+\partial^{\nu} u^{\mu}-u^{\mu} u^{\lambda} \partial_{\lambda} u^{\nu}-u^{\nu} u^{\lambda} \partial_{\lambda} u^{\mu}\right] \\
& +\left(\zeta-\frac{2}{3} \eta\right)\left[g^{\mu \nu}-u^{\mu} u^{\nu}\right] \partial_{\lambda} u^{\lambda}
\end{aligned}
$$

gives the first-order viscous correction with the bulk viscosity $\zeta$ and the shear viscosity $\eta$.

In order to determine these transport coefficients in kinetic theory one exploits the fact that $T^{\mu \nu}$ is related to the phase-space distribution functions $f(x, p) \equiv f(\mathbf{x}, \mathbf{p}, t)$ of the particles involved. In the present study we limit ourselves to a gas of pions. Then we have

$$
T^{\mu \nu}(x)=g_{\pi} \int \frac{\mathrm{d}^{3} p}{(2 \pi)^{3} E} p^{\mu} p^{\nu} f(x, p),
$$

where $g_{\pi}=3$ is the isospin degeneracy of the pions and $p^{0} \equiv E=\sqrt{m_{\pi}^{2}+\mathbf{p}^{2}}$ is their on-shell energy. In a relativistic quantum mechanical framework, the space-time evolution of the distribution function is governed by the Boltzmann-Uehling-Uhlenbeck (BUU) equation

$$
\frac{p^{\mu}}{E} \partial_{\mu} f(x, p)=C_{\pi \pi}[f],
$$

where the collision term $C_{\pi \pi}[f]$ encodes the changes of the distribution function due to interaction processes. In the following we restrict ourselves to $2 \rightarrow 2$ processes, $p+p_{1} \leftrightarrow p^{\prime}+p_{1}^{\prime}$. Then the collision term is given by

$$
\begin{aligned}
C_{\pi \pi}\left[f_{\pi}\right]= & \frac{g_{\pi}}{2} \int \frac{\mathrm{d}^{3} p^{\prime}}{(2 \pi)^{3}} \int \frac{\mathrm{d}^{3} p_{1}}{(2 \pi)^{3}} \int \frac{\mathrm{d}^{3} p_{1}^{\prime}}{(2 \pi)^{3}} \times \\
\times & \left\{\left|\mathcal{M}_{\pi \pi}\right|^{2} \frac{(2 \pi)^{4} \delta^{4}\left(p+p_{1}-p^{\prime}-p_{1}^{\prime}\right)}{16 E E_{1} E^{\prime} E_{1}^{\prime}} \times\right. \\
& \times\left[f_{p^{\prime}} f_{p_{1}^{\prime}}\left(1+f_{p}\right)\left(1+f_{p_{1}}\right)\right. \\
& \left.\left.-f_{p} f_{p_{1}}\left(1+f_{p^{\prime}}\right)\left(1+f_{p_{1}^{\prime}}\right)\right]\right\}
\end{aligned}
$$

where we have introduced the short-hand notation $f_{p} \equiv$ $f(x, p)$. The main physics input is the square of the isospin averaged invariant matrix element for $\pi \pi$ scattering:

$$
\left|\mathcal{M}_{\pi \pi}\right|^{2} \equiv \frac{1}{9} \sum_{I=0}^{2}(2 I+1)\left|\mathcal{M}_{\pi \pi}^{I}\right|^{2},
$$

where $\mathcal{M}_{\pi \pi}^{I}$ corresponds to the isospin- $I$ channel. These matrix elements will be evaluated in sect. [II] within the NJL model.

For a local Bose distribution with temperature $T$ and pion chemical potential $\mu_{\pi}$,

$$
f_{p}^{(0)}=\frac{1}{\exp \left[\left(p^{\nu} u_{\nu}(x)-\mu_{\pi}(x)\right) / T(x)\right]-1}
$$


the collision term vanishes, i.e., $C_{\pi \pi}\left[f^{(0)}\right]=0$. Because of this, the system is then said to be in 'local thermal equilibrium'. In general, however, $f_{p}^{(0)}$ is not a solution of the BUU equation, since the derivatives on the lefthand side of eq. (5) do not vanish for $x$-dependent fields.

We are particularly interested in static solutions ${ }^{1}$ with a non-vanishing shear flow. To this end, we work in the local rest frame of the fluid, $u=(1,0,0,0)^{T}$, and consider a time-independent equilibrium distribution $f^{(0)}$ with $\partial^{i} u^{j} \neq 0$ for some spatial indices $i \neq j$. Moreover, we assume that $T$ and $\mu_{\pi}$ do not depend on $x$. The solution of the the BUU equation is then constructed in the framework of a Chapman-Enskog expansion 35 37 to leading order. This means, we write

$$
f_{p}=f_{p}^{(0)}+f_{p}^{(1)},
$$

and linearize the collision term $C^{\pi \pi}$ in the correction $f_{p}^{(1)}$, while the advective term (the left-hand side of eq. (5) is evaluated with $f_{p}^{(0)}$ only. Parametrizing $f_{p}^{(1)}$ as

$$
f_{p}^{(1)}=f_{p}^{(0)}\left(1+f_{p}^{(0)}\right) B_{p}^{i j} \tau_{i j}^{\text {shear }}
$$

where $\tau_{i j}^{\text {shear }}=\frac{1}{2}\left(\partial_{i} u_{j}+\partial_{j} u_{i}-\frac{2}{3} \delta_{i j} \vec{\nabla} \cdot \vec{u}\right)$ in the local rest frame of the fluid, one finally arrives at

$$
\begin{aligned}
&-f_{p}^{(0)}(1\left.+f_{p}^{(0)}\right) \frac{p^{i} p^{j}}{E T}= \\
& \frac{g_{\pi}}{2} \int \frac{\mathrm{d}^{3} p^{\prime}}{(2 \pi)^{3}} \int \frac{\mathrm{d}^{3} p_{1}}{(2 \pi)^{3}} \int \frac{\mathrm{d}^{3} p_{1}^{\prime}}{(2 \pi)^{3}}(2 \pi)^{4} \delta\left({ }^{4} p+p_{1}-p^{\prime}-p_{1}^{\prime}\right) \times \\
& \quad \times \frac{\left|\mathcal{M}_{\pi \pi}\right|^{2}}{16 E E_{1} E^{\prime} E_{1}^{\prime}} f_{p}^{(0)} f_{p_{1}}^{(0)}\left(1+f_{p^{\prime}}^{(0)}\right)\left(1+f_{p_{1}^{\prime}}^{(0)}\right) \times \\
& \times\left[B_{p^{\prime}}^{i j}+B_{p_{1}^{\prime}}^{i j}-B_{p}^{i j}-B_{p_{1}}^{i j}\right],
\end{aligned}
$$

which is a linear integral equation for the tensor function

$$
B_{p}^{i j}=\mathcal{B}(|\vec{p}|)\left(\hat{p}^{i} \hat{p}^{j}-\frac{1}{3} \delta^{i j}\right),
$$

where $\hat{p}=\vec{p} /|\vec{p}|$. We solve this equation by expanding the function $\mathcal{B}$ in generalized Sonine polynomials. Further details are given in the appendix.

Having constructed the distribution function $f$, we can now insert it into eq. (4) to determine the energymomentum tensor. The shear viscosity is then obtained

1 Since we want to calculate static transport coefficients, we should consider static solutions. In principle, we can set up the system to be in local thermal equilibrium at some given time $t_{0}$. However, the BUU equation then drives the system away from the equilibrium distribution, i.e., this solution would not be static. by comparing the result with Eqs. (1) - (3). In fact, as the equilibrium distribution just gives rise to the ideal part of $T^{\mu \nu}$, the relevant contribution comes from the correction term $f^{(1)}$. One finds

$$
\eta=\frac{g_{\pi}}{15} \frac{4 \pi}{(2 \pi)^{3}} \int_{0}^{\infty} \mathrm{d} p \frac{p^{4}}{E} f_{p}^{(0)}\left(1+f_{p}^{(0)}\right) \mathcal{B}(p) .
$$

Finally we note that the kinetic description is only valid in the dilute-gas limit, i.e., if the system is well approximated by a free gas and interactions lead only to minor corrections. This means that the mean free path $\lambda$ has to be much larger than the typical range of interaction $r$. To assess the region of validity of our results we therefore have to check whether this condition is fulfilled.

\section{IN-MEDIUM PION SCATTERING IN THE NJL MODEL}

The remaining task for the calculation of the shear viscosity in the approximation, discussed above, is the determination of the in-medium pion mass $m_{\pi}$ and the $\pi \pi$ scattering amplitude $\mathcal{M}_{\pi \pi}$. As motivated in the Introduction, this will be done within the NJL model, which has the advantage that it allows for calculating mediumdependent meson properties, including effects of their quark substructure and the chiral transition.

In the present analysis we restrict ourselves to two quark flavors and use the standard NJL-model Lagrangian [24, 25],

$$
\mathcal{L}=\bar{\psi}\left(i \not \partial-m_{0}\right) \psi+g\left[(\bar{\psi} \psi)^{2}+\left(\bar{\psi} i \gamma_{5} \vec{\tau} \psi\right)^{2}\right],
$$

with four-point interactions in the scalar-isoscalar $(\sigma)$ and pseudoscalar-isovector $(\pi)$ channels. Here $\psi$ denotes a quark field, $m_{0}$ is the bare quark mass, and $g$ is a coupling constant with mass dimension -2 . The model is not renormalizable and we use a 3 -momentum cut-off $\Lambda$ in order to regularize divergent loop integrals. For the numerical calculations we take the parameters $m_{0}=5.0 \mathrm{MeV}$, $g \Lambda^{2}=2.06$, and $\Lambda=664.3 \mathrm{MeV}$ from [29].

\section{A. Pion mass}

The strong attractive interaction between the quarks leads to the dynamical generation of a mass gap. In the Hartree approximation, this so-called constituent quark mass is given by

$$
m_{q}=m_{0}+\Sigma_{H}
$$




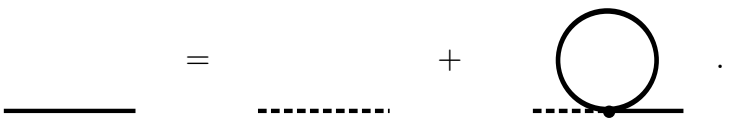

FIG. 1: Gap equation in the Hartree approximation. The dashed line corresponds to the bare quark propagator $S_{0}$, the solid line corresponds to the dressed quark propagator $S$.

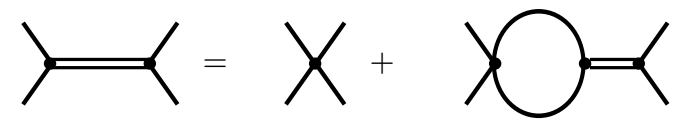

FIG. 2: Bethe-Salpeter equation for quark-antiquark scattering in Random-Phase Approximation. The T-matrix is depicted by double lines, while single lines denote the dressed quark propagator.

where $\Sigma_{H}$ is the one-loop self-energy, diagrammatically depicted in fig. 1 We refer to the literature for the exact expression of $\Sigma_{H}$, see e.g. 38] for details. Note that unlike the bare mass $m_{0}, \Sigma_{H}$ and, thus, $m_{q}$ are temperature dependent, so that typically $m_{q} \gg m_{0}$ at low $T$, whereas $m_{q} \approx m_{0}$ at high $T$.

Quark-antiquark scattering processes in specific channels can be associated with the propagation of a meson. In the Random-Phase Approximation (RPA) the BetheSalpeter equation for the quark-antiquark $T$-matrix takes the form, diagrammatically shown in fig. 2 This approximation is a standard technique in the NJL model, again we refer to [38] for details of the calculation. Evaluation of this equation in the scalar and pseudoscalar channels, respectively, yields the meson "propagators"

$$
D_{M}^{R P A}(q)=\frac{-2 g}{1-2 g \Pi_{M}^{R P A}(q)} .
$$

where $M \in\left\{\pi^{a}, \sigma\right\}$, and $\Pi_{M}$ denotes the corresponding quark-antiquark polarization loop. For the momenta we have used the short-hand notation $q \equiv\left(i \omega_{m}, \vec{q}\right)$ with bosonic Matsubara frequencies $\omega_{m}=2 m \pi T$. After analytic continuation to real energies, which simply amounts to replacing $i \omega_{m}$ by $q_{0}+i \varepsilon$ in the final expressions, ${ }^{2}$ the meson masses $m_{M}$ can be extracted from the position of

\footnotetext{
2 This corresponds to the retarded propagator. Alternatively, we could replace $i \omega_{m}$ by $q_{0}+i \operatorname{sign}\left(q_{0}\right) \varepsilon$ for the time-ordered propagator.
}

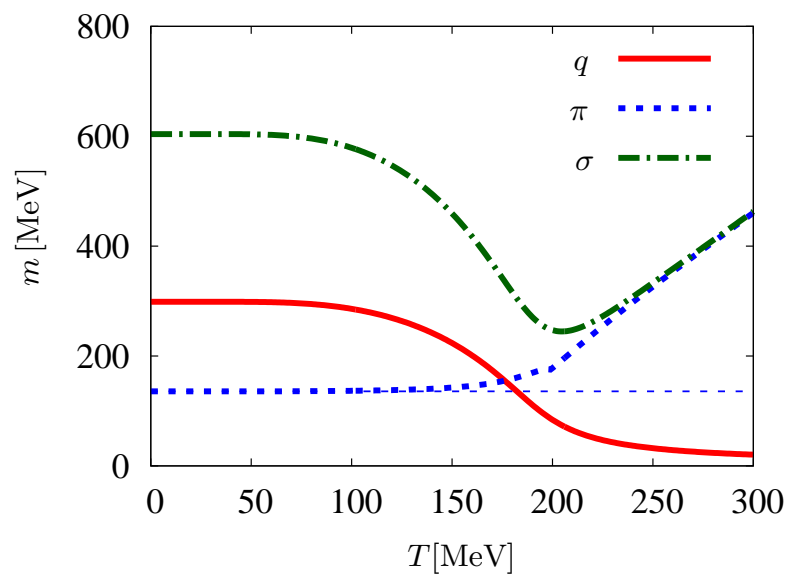

FIG. 3: Masses of constituent quarks (solid/red line), pions (dotted/blue line), and $\sigma$-mesons (dashed-dotted/green line) as functions of temperature. The vacuum-pion mass is shown as a thin line.

the pole of $D_{M}$,

$$
1-2 g \operatorname{Re} \Pi_{M}^{R P A}\left(q_{0}=m_{M}, \overrightarrow{0}\right)=0 .
$$

Here we have chosen to define the "mass" of an unbound resonance via the real part of $\Pi_{M}$, while for bound-state solutions, i.e., for $q_{0}=m_{M}<2 m_{q}$ the polarization function is real anyway.

Like the constituent quark mass, the masses of the pion and the $\sigma$-meson are temperature dependent. This is well known from the literature (see, e.g., 27]) and shown in fig. 3 for our choice of parameters. The latter have been fitted to reproduce a pion mass of $m_{\pi}=135 \mathrm{MeV}$ in vacuum [29]. In the chirally broken phase, the pion is protected by being an approximate Goldstone boson, and hence the temperature dependence of $m_{\pi}$ is very weak. The mass of the $\sigma$-meson, on the other hand, is approximately given by $2 m_{q}$ and therefore drops considerably when approaching the crossover temperature. In the restored phase, $m_{\pi}$ and $m_{\sigma}$ rise again and become asymptotically degenerate.

For the later discussion of the $\pi \pi$-scattering cross section it is useful to define two characteristic temperatures in the crossover region, which have been introduced first in ref. [32]. The first one is the $\sigma$-dissociation temperature, defined by

$$
m_{\sigma}\left(T_{d i s s}\right)=2 m_{\pi}\left(T_{d i s s}\right) .
$$

For $T<T_{\text {diss }}$, the $\sigma$-meson can decay into two pions. The second is the Mott-temperature, defined by

$$
m_{\pi}\left(T_{M o t t}\right)=2 m_{q}\left(T_{M o t t}\right) .
$$



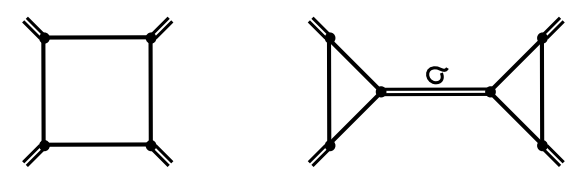

FIG. 4: Diagrams contributing to $\pi \pi$-scattering in leadingorder $1 / N_{c}$. Left: box diagram. Right: $\sigma$-meson exchange. In the quark loops, both directions of the momentum flow should be taken into account.

For $T>T_{M o t t}$, the pion can decay into a quark-antiquark pair, i.e., it is no longer a bound state but only a $q \bar{q}$ resonance. With the parameter set chosen here, we have $T_{\text {diss }}=179.9 \mathrm{MeV}$ and $T_{\text {Mott }}=198.55 \mathrm{MeV}$. In the chiral limit, both temperatures are equal to the critical temperature of the chiral phase transition. Thus, they can be taken as possible definitions of the chiral crossover temperature.

\section{B. $\pi \pi$-scattering length}

Since pions are not elementary degrees of freedom in the NJL model, there is no elementary pion-pion interaction in the model. However, effective meson-meson vertices can be constructed by using the diagrammatic building-blocks of the NJL model. We follow [31, 32] and consider the leading-order diagrams in a $1 / N_{c}$-expansion. The two diagrams contributing to $\mathcal{M}_{\pi \pi}$ to that order are shown in fig. 4. In the first diagram the pions are coupled through an intermediate quark loop ('box'), while the second corresponds to the exchange of a $\sigma$-meson, which is coupled to the external pions via quark triangles. $^{3}$ To be precise, the diagrams shown in fig. 4 are representatives of two classes of diagrams which must be considered [31]. In the case of the box diagram, the pions can be attached to the quark loop in various orderings, leading to three distinct amplitudes. Moreover, the $\sigma$-meson exchange must be taken in the $s^{-}, t$ - and $u$-channel. These contributions can be coupled to the different isospin channels. The $\pi \pi$-scattering length is

3 In the limit of infinitely heavy quarks, the quark box and the triangle shrink to a pointlike four-pion and $\sigma \pi \pi$-vertex, respectively. The diagrams shown in fig. 4 then reduce to the leading-order contributions for $\pi \pi$-scattering in the linear sigma model [39].

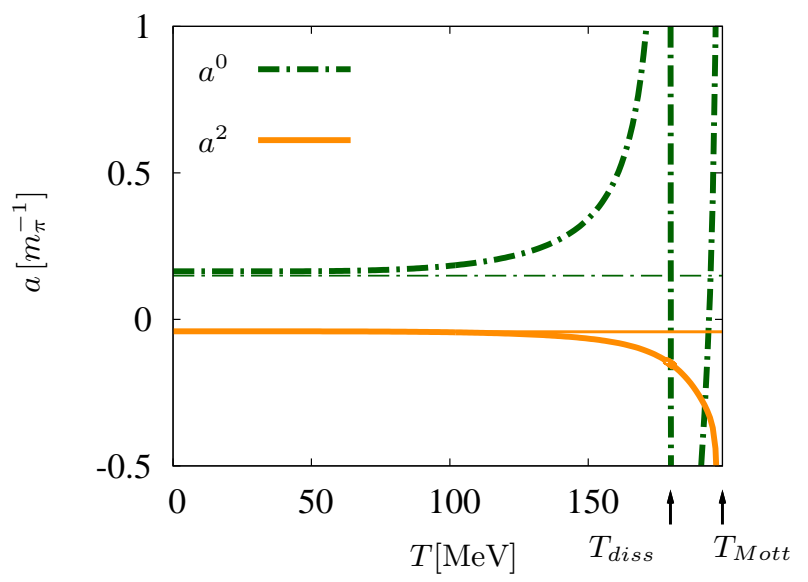

FIG. 5: The $\pi \pi$-scattering lengths as functions of temperature. The dash-dotted (green) lines correspond to $I=$ 0 , whereas the solid (orange) lines correspond to $I=2$. Bold lines denote the NJL results, whereas the thin lines correspond to the vacuum-extrapolated Weinberg scattering lengths.

related to the invariant matrix element at threshold, ${ }^{4}$

$$
a^{I}=\frac{1}{32 \pi m_{\pi}} \mathcal{M}_{\pi \pi}^{I}\left(s=4 m_{\pi}^{2}, t=u=0\right) .
$$

The explicit expressions for $a^{0}$ and $a^{2}$ as functions of temperature are given in ref. [32], while $a^{1}$ vanishes, as it should for $s$-wave scattering.

The results are shown in fig. 5. With the parameter set used in this article, we find the same qualitative behavior as in 32]. The vacuum values $a^{0}=0.164 m_{\pi}^{-1}$ and $a^{2}=-0.041 m_{\pi}^{-1}$, are in reasonable agreement with the Weinberg scattering lengths [40],

$$
a_{W}^{0}=\frac{7 m_{\pi}}{32 \pi f_{\pi}^{2}}, \quad a_{W}^{2}=-\frac{2 m_{\pi}}{32 \pi f_{\pi}^{2}} .
$$

which yield $a_{W}^{0}=0.149 m_{\pi}^{-1}$ and $a_{W}^{2}=-0.0425 m_{\pi}^{-1}$. Note that the chiral expansion employed by Weinberg is not identical to the $1 / N_{c}$ expansion. Thus perfect agreement should not be expected.

At finite temperature, the scattering lengths deviate from their vacuum values. This effect is very small at low temperatures but becomes important when approaching the crossover region, in particular the two transition temperatures $T_{\text {diss }}$ and $T_{\text {Mott }}$. At $T=T_{\text {diss }}$, the process

\footnotetext{
4 Since we do not expect any confusion, we denote both, the center-of momentum energy and the entropy density, by $s$, as customary.
} 
$\pi+\pi \rightarrow \sigma$ is resonant for two pions at rest. Thus the $s$-channel $\sigma$-exchange diagram involves an on-shell $\sigma$ propagator, giving rise to a strong peak in the isoscalar channel. ${ }^{5}$ Similarly, at $T=T_{\text {Mott }}$, a pion at rest is at threshold for the decay process into a quark-antiquark pair. This leads to a divergent scattering length in both isospin channels at the Mott temperature

The resonant scattering length at $T_{\text {diss }}$ and $T_{M o t t}$ is reminiscent of the behavior of cold atomic gases in an external magnetic field in the vicinity of a Feshbach resonance. In the present case the role of the magnetic field is taken by the temperature. This analogy between cold atoms and hot pions will be investigated further in the following sections.

\section{Scattering amplitude}

As discussed in sect. II the calculation of the shear viscosity of an interacting pion gas within kinetic theory involves the in-medium scattering amplitudes $\mathcal{M}_{\pi \pi}^{I}$. In the following we introduce four approximate ways to evaluate $\mathcal{M}_{\pi \pi}^{I}$. Comparison of the corresponding results will then allow to identify the most relevant effects.

\section{Weinberg amplitude}

The most simple approximation is to neglect both, momentum and temperature dependence of the scattering amplitude, and to employ Eqs. (20) and (21) to construct the latter from the Weinberg scattering lengths,

$$
\mathcal{M}_{\pi \pi, W}^{0}=\frac{7 m_{\pi}^{2}}{f_{\pi}^{2}}, \quad \mathcal{M}_{\pi \pi, W}^{2}=-\frac{2 m_{\pi}^{2}}{f_{\pi}^{2}}
$$

This approximation has previously been discussed in ref. [13].

\section{Medium modified momentum independent amplitude}

We have seen in sect. IIIB that the assumption of a temperature independent scattering amplitude is definitely not a good approximation when approaching the crossover region. Therefore, in order to improve on this,

\footnotetext{
${ }^{5}$ Note that $a^{0}$ does not diverge at $T=T_{\text {diss }}$ since in the NJL model the $\sigma$-meson has a small but nonvanishing width due to $q \bar{q}$-decay.
}

we replace the Weinberg scattering lengths by the temperature dependent scattering lengths of fig. 5. However, we still neglect the momentum dependence, i.e., we evaluate the amplitudes only at the two-pion threshold,

$$
\mathcal{M}_{\pi \pi, t h}^{I}=\mathcal{M}_{\pi \pi}^{I}\left(s=4 m_{\pi}^{2}, t=u=0\right) .
$$

At low temperature the Weinberg amplitudes are approximately recovered because of the good agreement of the NJL scattering lengths with the Weinberg ones. On the other hand, $\mathcal{M}_{\pi \pi, t h}^{I}$ also incorporates the "Feshbach resonances" at $T_{\text {diss }}$ and $T_{\text {Mott }}$. Of course, this will be important for the shear viscosity.

\section{Momentum dependent $\sigma$-meson exchange}

On the other hand, in the crossover region where the temperature is of the order of the pion mass, the approximation of a generally momentum dependent scattering amplitude by its value at the two-pion threshold becomes questionable as well. This is most obvious for the $\sigma$-meson exchange diagram (Fig. 4, right) in the $s$ channel. As explained above, the resonant behavior of $a^{0}$ at $T=T_{\text {diss }}$ is due to the fact that at this temperature the $\sigma$-meson propagator is on-shell for pions at rest. However, when the thermal motion of the pions is taken into account, the $\sigma$-meson can become on-shell already at lower temperatures, while at $T=T_{\text {diss }}$ a large fraction of pion pairs is far away from the threshold.

In order to include this effect, we again improve our approximation scheme and take the momentum dependence of the $\sigma$-propagator into account. To be precise, in the $s$-channel we consider a $\sigma$-meson with energy $q^{0}=\sqrt{s}$ and vanishing three-momentum, while in the $t$ - and $u$ channel we take $q^{0}=0$ and $|\vec{q}|=t$ or $u$, respectively. For simplicity, we still neglect the momentum dependence of the quark triangle and box diagrams. This approximation is sometimes called the 'static limit' and can be interpreted as not resolving the quark substructure of the effective meson-meson vertices. We expect that this is a minor effect, except for temperatures close to and above $T_{\text {Mott }}$.

For small momenta, the resulting scattering amplitude is equal to $\mathcal{M}_{\pi \pi, t h}^{I}$, so the Weinberg scattering length is again well reproduced at $T=0$. Above the two-pion threshold there is now also a small $p$-wave contribution, giving rise to a non-vanishing amplitude in the isospin-1 channel. This is taken into account in our calculations. However, for a realistic description of this channel, the 


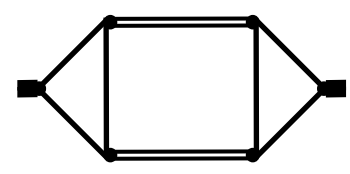

FIG. 6: Selfenergy correction to the $\sigma$-meson propagator with two intermediate pions.

$\rho$-meson has to be included. This extention of the model is left for future work.

\section{Including the $\pi \pi$-decay width of the $\sigma$-meson}

A still unrealistic feature of the previous approximation is the structure of the intermediate $\sigma$-meson. While the process $\pi \pi \leftrightarrow \sigma$ is an essential ingredient of the $\pi \pi$-scattering amplitude, the coupling of the $\sigma$-meson to a two-pion intermediate state is not included in the RPA Bethe-Salpeter equation, depicted in fig. 2. As a consequence, the $\sigma$-meson only has a small width due to quark-antiquark decay, whereas the $\pi \pi$-decay width, which should be dominant at low temperature, is not taken into account.

Thus, in an improved approximation we consider a dressed $\sigma$-propagator,

$$
D_{\sigma}^{\text {dressed }}(q)=\frac{-2 g}{1-2 g \Pi_{\sigma}^{\text {dressed }}(q)},
$$

where $\Pi_{\sigma}^{\text {dressed }}(q)$ is a sum of the RPA polarization loop and a correction term which contains a two-pion intermediate state. This correction can be derived systematically in an expansion in the inverse number of colors $\left(1 / N_{c}\right)$ to next-to-leading order [38, 41 46]. The relevant diagram is shown in fig. 6 and yields

$$
\Pi_{\sigma}^{\pi \pi}(q)=-\frac{3}{2} \Gamma_{\sigma \pi \pi}^{2} T \sum_{n} \int \frac{d^{3} k}{(2 \pi)^{3}} D_{\pi}^{R P A}(q+k) D_{\pi}^{R P A}(k),
$$

with $q=\left(i \omega_{m}, \vec{q}\right), k=\left(i \omega_{n}, \vec{k}\right)$ and bosonic Matsubara frequencies $\omega_{m}$ and $\omega_{n} . \Gamma_{\sigma \pi \pi}$ denotes the effective $\sigma \pi \pi$ vertices, i.e., the quark triangles in fig. 6] which are again evaluated in the static limit.

After analytical continuation to real energies, $\Pi_{\sigma}^{\pi \pi}$ has a nonvanishing imaginary part above the two-pion threshold. This is exactly the wanted effect. In addition, it has also a real part, leading to a renormalization of the $\sigma$-meson mass. Unfortunately, this causes inconsistencies with chiral low-energy theorems, which are based on a delicate cancellation between the $\sigma$-exchange and the box diagram in fig. 4 .

This problem cannot easily be cured. In principle, consistency with chiral symmetry can be achieved in a systematic $1 / N_{c}$ expansion by taking into account all diagrams of a given order. At leading order these are the diagrams shown in fig. 4 with the RPA $\sigma$-meson, while at next-to-leading order there are more than 60 additional diagrams, not counting the different orderings to attach the external pions. Moreover, the fact that fig. 6 is iterated in the $\sigma$-propagator spoils the strict $1 / N_{c^{-}}$-counting, and we would encounter inconsistencies even if all nextto-leading order diagrams were considered.

In this situation, we decided to include the $\sigma$-width in a minimal fashion by considering only the imaginary part of $\Pi_{\sigma}^{\pi \pi}$ but neglecting the real part, i.e., we take

$$
\Pi^{\text {dressed }}(q)=\Pi_{\sigma}^{R P A}(q)+i \operatorname{Im} \Pi_{\sigma}^{\pi \pi}(q) .
$$

As a consequence, the propagator is not modified at and below the two-pion threshold, which implies that the scattering lengths remain unchanged. In particular, the Weinberg scattering lengths are still reproduced well at $T=0$.

Of course, this way of including the $\sigma$-width is very schematic. At the present stage we mainly want to estimate its importance for the shear viscosity, while we are not aiming a perfect description of $\pi \pi$-scattering. We note that neglecting the real part of $\Pi_{\sigma}^{\pi \pi}$, which is related to the imaginary part by the Kramers-Kronig relation, violates causality. We believe, however, that this is a minor effect in comparison with the violation of the chiral low-energy theorems, which would occur if the real part was taken into account.

In fig. 7 , the spectrum of the $\sigma$-meson is shown for different temperatures. The "spectral function" 6

$$
\rho_{\sigma}(q)=-2 \operatorname{Im} D_{\sigma}(q)
$$

is shown for $|\vec{q}|=0$ as a function of energy for both approximations, RPA as well as the additional dressing. In the vacuum, the RPA meson has a very small spectral width, while the dressed $\sigma$-meson is much broader. When

\footnotetext{
6 The meson propagators as defined in Eqs. (16) and (24), and, thus, the spectral functions defined in eq. (27) are not properly normalized. However, since it is the unrenormalized $\sigma$ propagator which enters into the $\pi \pi$-scattering matrix elements, we prefer to compare the corresponding unnormalized spectral functions in fig. 7
} 


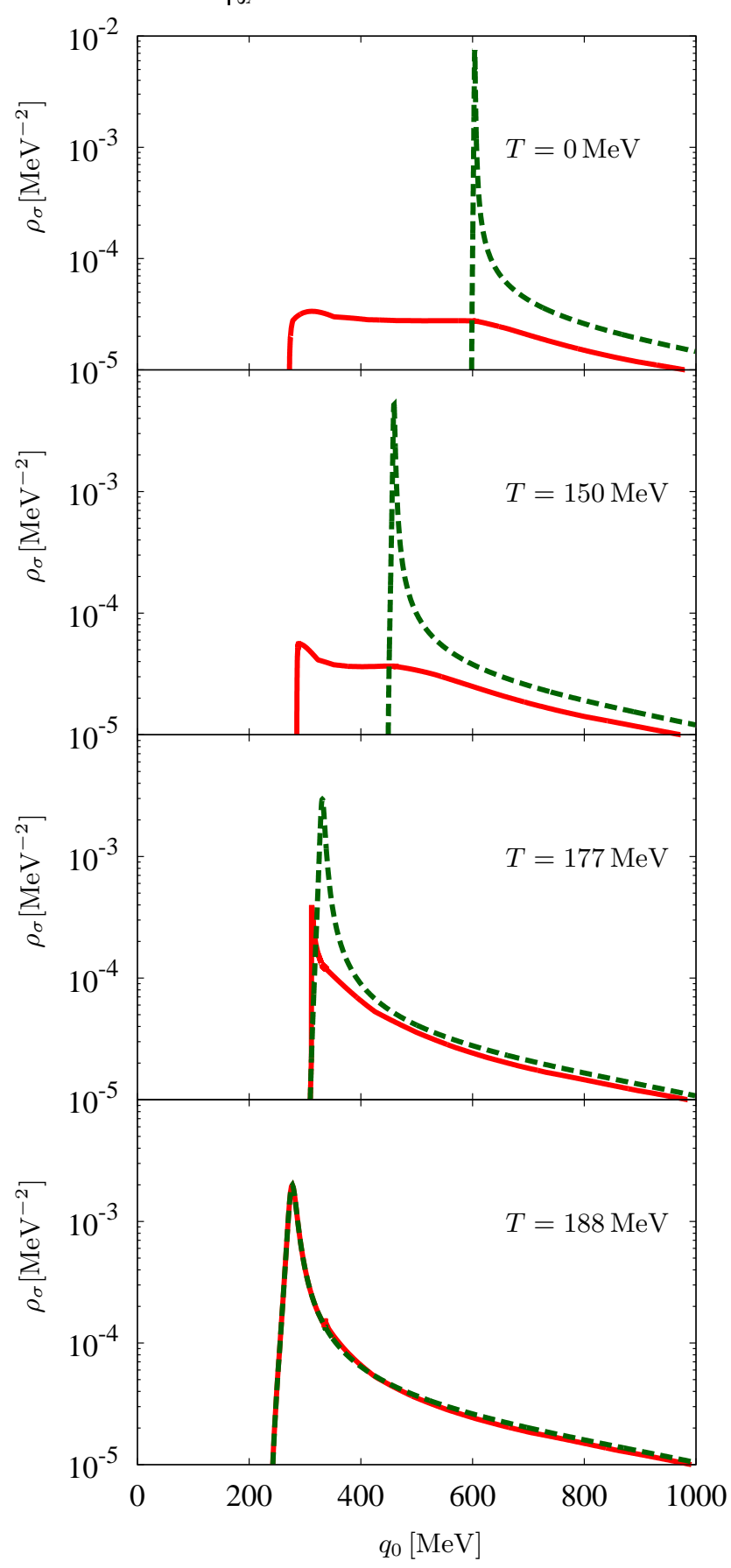

FIG. 7: Unnormalized spectral functions of the $\sigma$-meson as functions of energy $q_{0}$ for different temperatures. The dashed (green) lines indicate the RPA calculations, while the solid (red) lines include dressing of the $\sigma$-meson as described in the text.

the temperature increases, the quark mass decreases and thus the threshold for the RPA meson moves downwards. At the same time, the mass peak of the $\sigma$ moves downwards as well (see fig. 33). Therefore the phase space for the two-pion decay becomes smaller and the width of the dressed $\sigma$-meson gets reduced. At $T=177 \mathrm{MeV}$, which is near the dissociation temperature, the thresholds $2 m_{\pi}$ and $2 m_{q}$ are almost equal. At temperatures above the $T_{\text {diss }}$, the pion decay plays only a minor role, and the dressed $\sigma$-meson is very similar to the RPA result. The threshold in this temperature range is determined by the $q \bar{q}$-decay channel, which is included in both descriptions of the $\sigma$-meson.

\section{Scattering cross section}

The isospin-averaged differential cross section in the center-of-momentum frame is given by

$$
\left(\frac{\mathrm{d} \sigma}{\mathrm{d} \Omega}\right)_{c m}=\frac{\left|\mathcal{M}_{\pi \pi}\right|^{2}}{64 \pi^{2} s},
$$

where $\left|\mathcal{M}_{\pi \pi}\right|^{2}$ is the isospin-averaged squared scattering amplitude as defined in eq. (7). In fig. 8 we compare the total cross sections of the four different approximations introduced in sect. IIIC at four different temperatures: vacuum $(T=0)$, an intermediate temperature $(T=150 \mathrm{MeV}), T=177 \mathrm{MeV}$, which is closely below the $\sigma$-meson dissociation temperature, and $T=188 \mathrm{MeV}$, which is between $T=T_{\text {diss }}$ and $T=T_{\text {Mott }}$.

For all temperatures, the three curves based on the NJL model coincide for $\sqrt{s} \rightarrow 2 m_{\pi}$, but are in general quite different above threshold. In vacuum, since the NJL scattering lengths are in good agreement with the Weinberg values, the cross sections obtained with the momentum independent amplitudes $\mathcal{M}_{\pi \pi, t h}^{I}$ and $\mathcal{M}_{\pi \pi, W}^{I}$ (purple dash-dotted line) are almost identical. Taking into account the momentum dependence of the $\sigma$-meson propagator in RPA (green dashed line) leads to a very pronounced peak at $m_{\sigma} \approx 600 \mathrm{MeV}$, which gets strongly broadened when the $\pi \pi$-decay width is included (red solid line).

While the Weinberg cross section is temperature independent by construction, the NJL results change when the temperature is altered. The main effects can be attributed to the temperature dependence of the $\sigma$-meson mass and, closely related to this, to the temperature dependence of the scattering lengths, cf. Figs. 3 and 5. When the scattering amplitude is approximated by its threshold value (blue dotted lines), the latter simply leads to a scaling of the vacuum result by an energy independent factor, which basically follows the behavior of the squared scattering lengths. In particular 


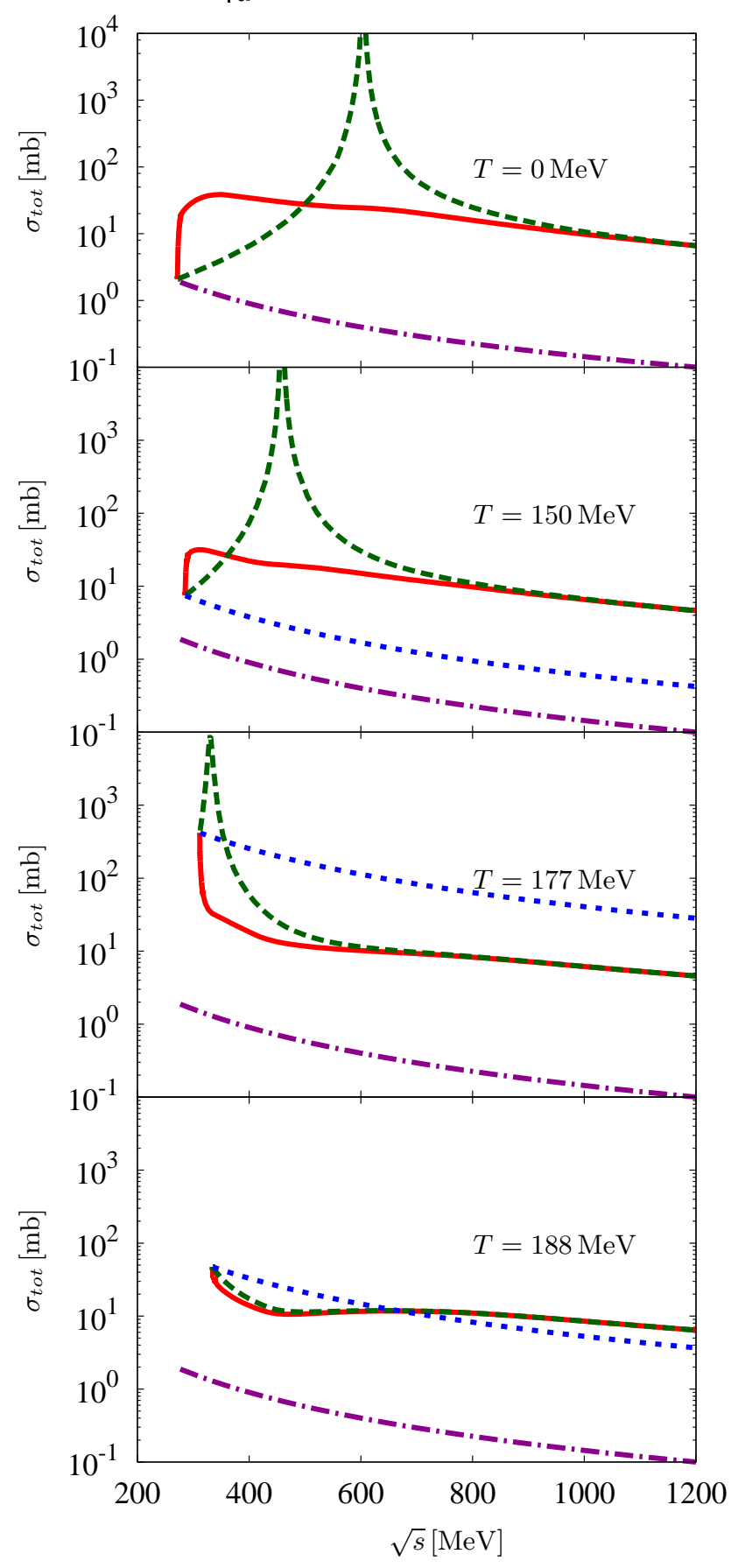

FIG. 8: Total cross section as a function of center-ofmomentum energy $\sqrt{s}$ for four different temperatures. The different curves correspond to different approximations. The dash-dotted (purple) lines were computed with the constant Weinberg scattering amplitudes $\mathcal{M}_{\pi \pi, W}^{I}$. The dotted (blue) lines correspond to the temperature-dependent but momentum-independent amplitudes $\mathcal{M}_{\pi \pi, t h}^{I}$. Note that this line coincides with the previous one in vacuum. For the results indicated by the dashed (green) lines, the momentum dependence of the $\sigma$-meson propagator was taken into account in RPA, while the solid (red) lines were computed with the dressed $\sigma$. the whole curve (almost) diverges at the "Feshbach resonances" $T=T_{\text {diss }}$ and $T=T_{\text {Mott }}$.

When the momentum dependence of the $\sigma$-meson propagator is taken into account, the temperature effects are more subtle. Since the $\sigma$-meson mass decreases, the corresponding peak in the RPA calculation moves downwards in energy (green dashed lines), reaches the threshold at $T=T_{\text {diss }}$ and finally moves out of the kinematically allowed regime. Thus, whereas at $T=150 \mathrm{MeV}$ the qualitative behavior is still similar to the vacuum case, at temperatures close to or above the $\sigma$-meson dissociation temperature, the cross sections can fall considerably below the approximation with the momentum independent amplitudes. It is also remarkable that, except for the threshold region, the cross sections obtained with the dressed $\sigma$-meson (red solid lines) are rather temperature independent.

Finally, we note that the NJL-model cross sections are typically orders of magnitude larger than the cross sections obtained from the Weinberg scattering lengths and agree with the latter only at low temperatures at threshold. Of course, this also has important consequences for the shear viscosity, which we discuss in the next section.

\section{SHEAR VISCOSITY AND THE IDEAL FLUID}

The masses and scattering amplitudes computed in the previous section can now be used to calculate the shear viscosity as outlined in sect. III, i.e., from Eq. (13). Thereby we restrict ourselves to the case of a vanishing pion chemical potential, $\mu_{\pi}=0$.

\section{A. Shear viscosity}

The shear viscosity $\eta$ as a function of temperature $T$ is shown in fig. 9, where again the results of the different approximations for the scattering amplitude are compared. For a qualitative interpretation it is useful to recall the simple nonrelativistic approximation

$$
\eta \approx \bar{p} /\left(3 \bar{\sigma}_{t o t}\right),
$$

where $\bar{p}$ and $\bar{\sigma}_{\text {tot }}$ are the thermally averaged pion momentum and cross section, respectively. Since with increasing temperature, increasing momenta and energies are probed, the cross section obtained with the constant Weinberg amplitude decreases with temperature (cf. fig. 8) and therefore the shear viscosity rises 

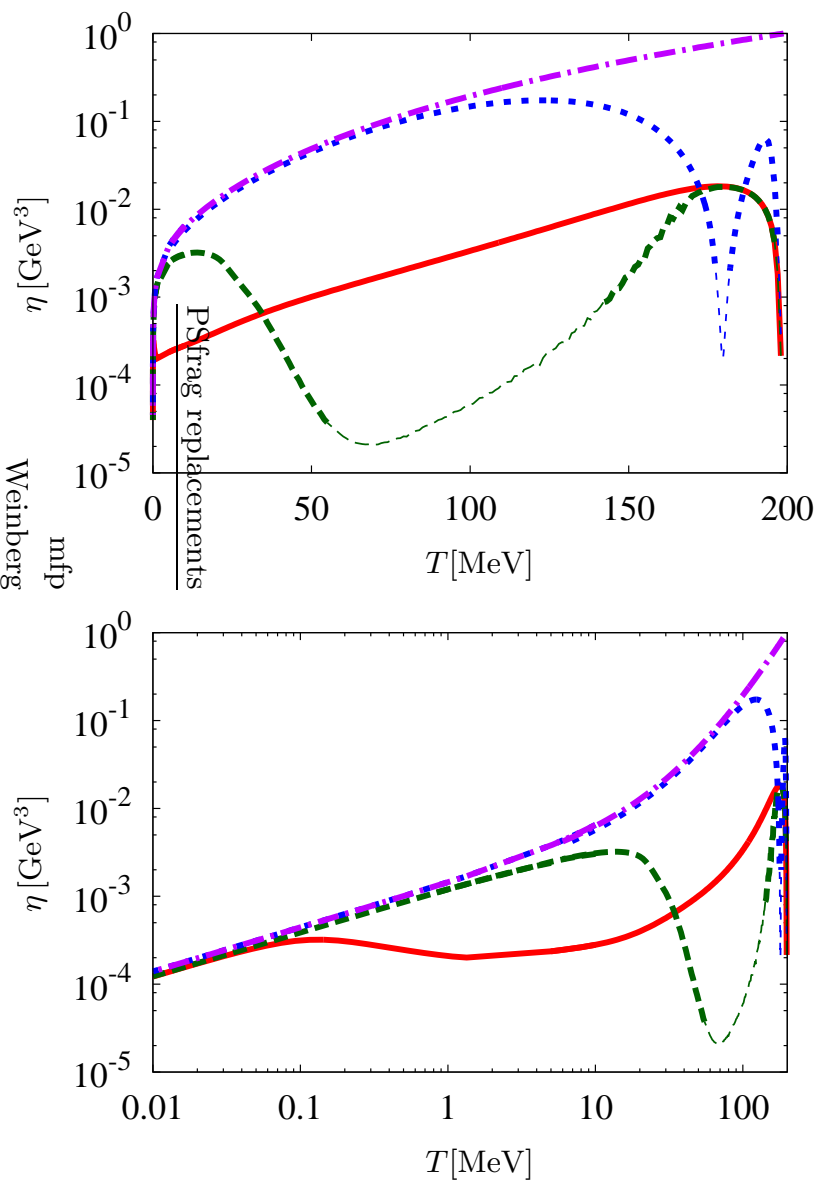

FIG. 9: The shear viscosity $\eta$ as a function of temperature for the different approximations for the scattering amplitudes discussed in the text. The lower part of the figure shows the same results as the upper part, but in a log-log representation in order to highlight the low-temperature behavior. The line styles are consistent with the choice in fig. 8 The dash-dotted (purple) line was obtained with the constant Weinberg scattering amplitudes $\mathcal{M}_{\pi \pi, W}^{I}$, the dotted (blue) line with the temperature dependent but momentum independent amplitudes $\mathcal{M}_{\pi \pi, t h}^{I}$. For the dashed (green) line, the momentum dependence of the $\sigma$-meson propagator was taken into account in RPA, while the solid (red) line was computed with the dressed $\sigma$. Thin lines indicate the regions where the validity of the Boltzmann approach is questionable. Here we have defined these regions as the intervals where the lower boundary of the corresponding band in fig. 10 is less than unity.

monotonously in this approximation (purple dash-dotted line).

Almost the same result is obtained with the momentum independent NJL amplitude (blue dotted line) in the regime below $T \approx 100 \mathrm{MeV}$, where this amplitude is in good agreement with the Weinberg value. On the other hand, in the vicinity of the chiral crossover, in particular at $T_{\text {diss }}$ and $T_{M o t t}$, the shear viscosity becomes very small because of the large cross section in this region.

When the momentum dependence of the RPA $\sigma$-meson propagator is taken into account (green dashed line), the minimum of the viscosity moves to lower temperature. In fact, we should expect that the minimum is roughly at the temperature where the mean center-of-momentum energy matches the $\sigma$-meson mass peak. This estimate yields $T_{\text {min }} \approx 100 \mathrm{MeV}$, while the exact value is even somewhat lower, $T_{\min } \approx 70 \mathrm{MeV}$. Accordingly, at low and intermediate temperatures, the shear viscosity is considerably lower than in the previous approximations. On the other hand, when the dissociation temperature is approached, the energy of most pion pairs is way above the $\sigma$-meson mass peak and, hence, the viscosity is much larger than in the case of the momentum independent threshold amplitude. Note, however, that the strong decrease towards the Mott temperature persists. This is most likely an artifact of neglecting the momentum dependence of the quark triangles and box diagrams.

When we consider the dressed $\sigma$-meson, we obtain the result indicated by the red solid line. At low temperature the broadening of the mass peak leads to a strong enhancement of the cross section in the relevant kinematical region, whereas at intermediate temperatures, it is strongly reduced. As a consequence, the dip which is found in RPA, leading to a minimum at $T \approx 70 \mathrm{MeV}$, is completely washed out. Above the dissociation temperature, the pion decay channel of the $\sigma$-meson is of course irrelevant, and the shear viscosity practically coincides with the RPA result.

Finally, it should be noted that in the low-temperature limit all approximations approach the Weinberg result. This follows from the fact that at low enough temperature the momenta of the pions can be neglected and therefore only the scattering lengths are relevant. Hence the viscosities obtained from the momentum dependent scattering amplitudes converge to the result from the momentum independent amplitude, which in turn is in good agreement with the Weinberg result. It turns out, however, that in particular for the dressed $\sigma$-meson one has to go to very low temperatures to see this behavior (see lower part of fig. 9). Here we find that, because of the steep rise of the cross section near the threshold (see fig. (8), the viscosity starts to deviate from the other approximations already at $T \approx 0.1 \mathrm{MeV}$. Above this value, 
it first decreases and reaches a minimum at $T \approx 2 \mathrm{MeV}$, while the other curves strongly grow in this interval. As a consequence, the viscosities differ already by about an order of magnitude at $T=5 \mathrm{MeV}$.

Of course, these low temperatures are only of conceptual interest. Since there is no pion chemical potential, the particle distribution functions decrease exponentially with decreasing $T$ and become extremely small. As a consequence we encountered numerical instabilities at temperatures below $5 \mathrm{MeV}$. In this temperature regime, we have therefore performed a nonrelativistic approximation in order to obtain numerically stable results.

\section{B. Validity of the kinetic description}

Although kinetic theory formally provides a viscosity for all interactions at any temperature, we should keep in mind that the underlying assumption is the dilute gas limit, i.e., the results can only be trusted if the mean free path $\lambda$ is much larger than the typical range of the interaction $r$. The mean free path is given by $\lambda=1 /\left(n \bar{\sigma}_{t o t}\right)$, where $n$ is the pion density, which can be calculated in the ideal-gas limit. For the thermally averaged cross section we simply invert eq. (29) and express it through the calculated shear viscosity. This yields $\lambda=3 \eta /(n \bar{p})$.

In addition, we have to estimate the interaction range in an appropriate way. Since in our model the long-range part of the $\pi \pi$-scattering is mediated by $\sigma$-meson exchange, we may set $r=1 / m_{\sigma}$. Two alternative estimates have been established in ref. [17]. The first is based on the hard-sphere limit, leading to $r=\sqrt{\bar{\sigma}_{t o t} / \pi}$. The second is the Compton wave length of the scattered particle, i.e., in our case $r=1 / m_{\pi}$.

Hence, since the definition of the interaction range is somewhat arbitrary, we check the validity of the kinetic approach by calculating $\lambda / r$ for all three estimates of $r$. The results agree qualitatively for a single scattering amplitude, but depend strongly on the scattering amplitude itself. This is shown in fig. 10, where the different bands indicate the regions between the smallest and the largest $\lambda / r$ for a given scattering amplitude. For the weakest interaction, the temperature and momentum independent Weinberg amplitude, the ratio $\lambda / r$ is much larger than unity for all temperatures of interest. For the other approximations, on the other hand, there are regions where $\lambda / r$ gets small and, thus, the Boltzmann approach is not valid. In fact, since $\lambda$ is proportional to $\eta$ in our estimate, these regions roughly coincide with the minima of the

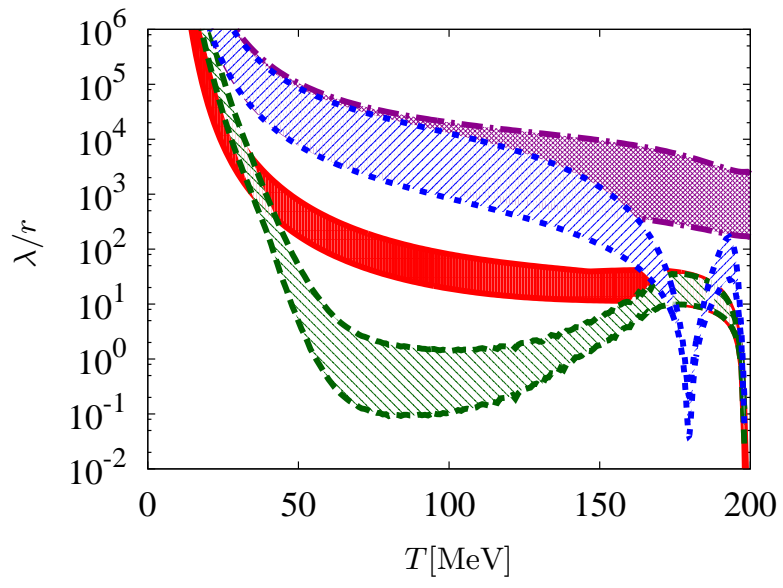

FIG. 10: The ratio $\lambda / r$ as a function of temperature for the different approximations for the scattering amplitudes. The bands indicate the region between the results obtained with the largest and the smallest estimate for $r$. The colors and the line styles bordering the shaded regions are consistent with the choice in Figs. 8 and 9 .

shear viscosity. For instance, for the momentum independent, but temperature dependent NJL-model amplitude, $\lambda / r$ gets very small in the vicinity of $T_{\text {diss }}$ and $T_{M o t t}$, whereas including the momentum dependence of the $\sigma$ propagator in RPA invalidates the BUU approach even at temperatures below $100 \mathrm{MeV}$. This is cured again, when the $\pi \pi$-decay width of the $\sigma$-meson is taken into account. In this case the results can be trusted until closely below the Mott temperature.

\section{Fluidity measures}

In order to compare the fluidity of systems with rather different natural scales, like cold atomic gases, water or the quark-gluon plasma, the absolute value of the shear viscosity is not very meaningful. More appropriate fluidity measures are therefore dimensionless ratios of $\eta$ and some thermal quantity, which scales in the proper way. The most prominent example, at least in the context of the quark-gluon plasma, is the ratio of shear viscosity and entropy density $s$, which has been conjectured to have a universal lower bound, $\eta / s \geq 1 / 4 \pi[33]$.

An alternative measure, which is better suited for the comparison of relativistic and nonrelativistic systems, has been suggested in ref. [47]. It is given by the ratio of 


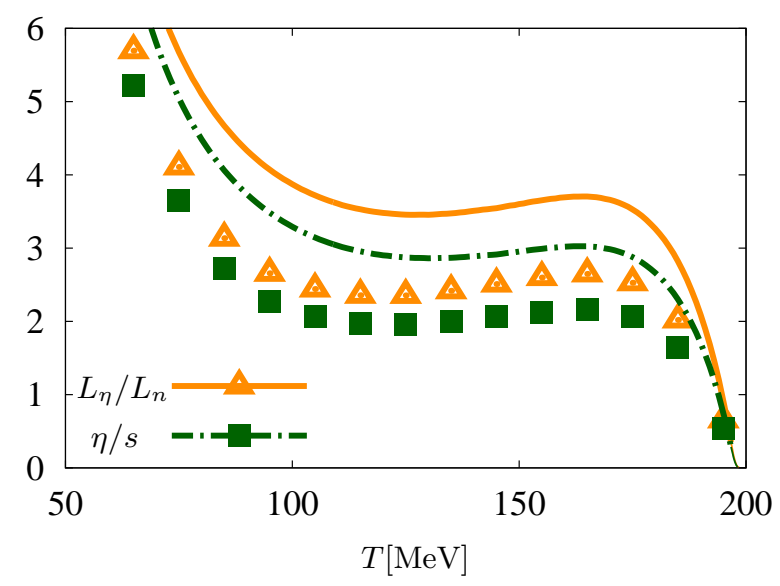

FIG. 11: The ratios $\eta / s$ (green dash-dotted line) and $L_{\eta} / L_{n}$ (orange solid line) as functions of temperature, calculated with the dressed $\sigma$-meson. The simple estimate $\eta \approx \bar{p} /(3 \sigma(\bar{p}))$ is indicated by triangles for $L_{\eta} / L_{n}$, and by squares for $\eta / s$.

two characteristic length scales

$$
L_{\eta} / L_{n}=\frac{\eta n^{1 / 3}}{h c_{s}}
$$

where $L_{\eta}=\eta /\left(h c_{s}\right)$ is related to the shear viscosity and $L_{n}=1 / n^{1 / 3}$ is related to the density. Here $n$ is the number density, $c_{s}$ is the sound velocity and $h=\epsilon+p$ is the enthalpy density.

Our results for $\eta / s$ and $L_{\eta} / L_{n}$ are shown in fig. 11] For the shear viscosity, we only take the most realistic amplitude, which includes the dressed $\sigma$-meson. The other quantities involved in the ratios, i.e., $s, n, h$ and $c_{s}$, are calculated in the ideal-gas limit, which is consistent with the Boltzmann approach. We find that both fluidity measures are very similar. After decreasing by several orders of magnitude at low temperatures, the curves become rather flat in an intermediate temperature regime, where we find $\eta / s \approx 3$ and a somewhat larger value for $L_{\eta} / L_{n}$. Finally, there is another steep decrease when $T$ approaches the Mott temperature. However, as already mentioned, this drop is probably an artifact of neglecting the momentum dependence of the quark triangles and boxes.

For comparison we also show the ratios one obtains when $\eta$ is calculated from the simple estimate eq. (29) with the additional assumption that the thermally averaged cross section $\bar{\sigma}_{t o t}$ can be approximated by the cross section at the averaged momentum $\sigma_{\text {tot }}(\bar{p})$. Compared with the Boltzmann approach, the results are about $30 \%$ lower but in good qualitative agreement. Hence, at a stage where the uncertainties in the scattering amplitude are still very large, this approximation is a viable alternative to the exact solutions of the BUU equations.

\section{DISCUSSION}

In this article, we have computed the shear viscosity of a pion gas within relativistic kinetic theory. Our main focus was to study the effects that originate from the chiral crossover transition. To this end we have employed a two-flavor NJL model to calculate the effective masses and scattering amplitudes of pions in a hot medium. The shear viscosity was then obtained from the solution of the BUU equation in a Chapman-Enskog expansion to leading order. Simultaneously we have carefully analyzed the range of validity of this approach, bearing in mind that kinetic theory can only be applied for dilute gases.

While at low temperatures our results are consistent with lowest-order chiral perturbation theory, the scattering of pions is strongly affected by the restoration of chiral symmetry at high temperature. In particular the lowering of the $\sigma$-mass leads to a strong enhancement of the $s$-channel $\sigma$-exchange diagram. As a consequence, the shear viscosity is much smaller than it would be expected from a simple vacuum extrapolation.

In the cross-over region the $\pi \pi$-scattering length diverges at two characteristic temperatures, $T_{\text {diss }}$ and $T_{M o t t}$. This is reminiscent of the situation in ultra-cold atomic gases in the vicinity of a Feshbach resonance. Motivated by this analogy, in a first step, we have neglected the momentum dependence of the scattering amplitude completely and only kept the temperature dependence according to the scattering length. Accordingly, the shear viscosity was found to have sharp minima at $T_{\text {diss }}$ and $T_{\text {Mott }}$ in this approximation.

On the other hand, unlike for cold atoms, the knowledge of the scattering length is not sufficient to describe the scattering of hot pions. Because of thermal motion, the $\sigma$-meson mass pole can be reached at temperatures well below the dissociation temperature, and we therefore find a considerable reduction of the shear viscosity at lower temperatures, when the momentum dependence of the scattering amplitude is taken into account.

However, the results turned out to be very sensitive to the approximations we have applied to evaluate the $\sigma$ propagator, and our 'most reliable' approximation, where we have included the $\sigma \rightarrow \pi \pi$-decay width, is surely not the last word. In fact, except for the region close to 
the Mott temperature, where the Boltzmann approach should not be trusted, our results for $\eta / s$ are still more than one order of magnitude above the conjectured lower bound of $1 / 4 \pi$. Thus, various improvements and extensions of the model should be performed:

The dressing of the $\sigma$-meson as described in sec. IIIC4 was done in a rather simple way by only including the imaginary part of the diagram shown in fig.6. As already pointed out, this ensures the correct threshold behavior, dictated by chiral symmetry, but it violates causality. One may try to cure this problem by calculating the real part of the of the diagram from a subtracted dispersion relation, with the subtraction constant chosen in such a way that the scattering lengths remain unchanged. This would be rather straightforward in the chiral limit. For physical pion masses it is more difficult because, even at threshold, the $\sigma$-meson in the $s$-channel is probed at a different kinematical point than in the $t$ - and $u$-channel ( $q^{2}=4 m_{\pi}^{2}$ and $q^{2}=0$, respectively), so that it is not immediately clear how to make the subtraction.

The dressed $\sigma$-meson can be viewed as to arise from iterating the $\sigma$-meson exchange diagram in fig. 4 In the same way one should also iterate the box diagram. Formally, this yields a series of diagrams which are of the same order in $1 / N_{c}$ as the dressing of the $\sigma$-meson and which contributes to the imaginary part above the twopion threshold as well. We have not considered these diagrams so far, because our primary interest was in getting rid of the sharp resonance peak caused by the RPA $\sigma$-meson. They might nevertheless give important contributions to the scattering amplitude. Taking them into account, makes it of course even more difficult to satisfy chiral theorems and causality, and one has to see whether dispersion techniques can help here as well. Maybe at that point one has to give up some of the formal requirements and should judge the reliability of the approximation by comparison with $\pi \pi$-scattering data at $T=0$.

In the $\pi \pi$-sector, we should also include intermediate $\rho$-mesons in order to obtain a realistic description of the $p$-wave isovector channel. In addition, we should include other hadrons, which are suppressed at low temperatures, but can become important in the crossover region 48 50]. In particular, we wish to extend the model to three flavors and include kaons and $\eta$-mesons. Moreover, we would like to include the scattering of quarks, which become important above the crossover temperature. On the other hand, in order to suppress quark effects in the hadronic phase, they should be coupled to the Polyakov loop. Work in these directions is in progress.

\section{Acknowledgments}

This work was supported in part by the Helmholtz International Center for FAIR, the Helmholtz Institute EMMI and the BMBF grant 06DA9047I.

\section{Appendix A: Generalized Sonine functions}

In order to solve the linear integral equation (11), we follow Refs. [13, 17] and expand the scalar function $\mathcal{B}(p)$ defined in eq. (12) in so-called generalized Sonine polynomials $P_{p}^{r} \equiv P^{r}(|\vec{p}|)$,

$$
\mathcal{B}(|\vec{p}|)=\sum_{r} b^{r} P_{p}^{r}
$$

The latter are polynomials of degree $r$,

$$
P_{p}^{r}=\sum_{j=0}^{r} c_{j}^{r} p^{j},
$$

which satisfy the orthogonality relations

$$
\int \mathrm{d} p \frac{p^{4}}{E_{p}} f_{p}^{(0)}\left(\left(1+f_{p}^{(0)}\right)\right) P_{p}^{r} P_{p}^{s}=\mathcal{N}^{r} \delta^{r s} .
$$

As in Refs. 13, 17] we do not choose the normalization constants $\mathcal{N}^{r}$ to be unity, but consider monic functions, $c_{r}^{r}=1$. The remaining coefficients $c_{j}^{r}$ are then uniquely determined by eq. (A3). These coefficients depend on $T$, $\mu_{\pi}$, and $m_{\pi}$, but the numerical orthogonalization is not a challenge.

Thus, the remaining problem is to compute the coefficients $b^{r}$ of the expansion (A1). To this end, we multiply both sides of eq. (11) with

$$
P^{r i j}(\vec{p})=P_{p}^{r}\left(\hat{p}^{i} \hat{p}^{j}-\frac{1}{3} \delta^{i j}\right)
$$

sum over the indices $i$ and $j$, and integrate over the momentum $\vec{p}$. Then, after inserting eq. (12) and the expansion (A1) for $B^{i j}$, one obtains a linear equation for the expansion coefficients,

$$
A^{r s} b^{s}=C^{r}
$$


with

$$
\begin{aligned}
& A^{r s}= \\
& \frac{g_{\pi}}{2} \int \frac{\mathrm{d}^{3} p}{(2 \pi)^{3}} \int \frac{\mathrm{d}^{3} p^{\prime}}{(2 \pi)^{3}} \int \frac{\mathrm{d}^{3} p_{1}}{(2 \pi)^{3}} \int \frac{\mathrm{d}^{3} p_{1}^{\prime}}{(2 \pi)^{3}} \times \\
& \quad \times(2 \pi)^{4} \delta^{4}\left(p+p_{1}-p^{\prime}-p_{1}^{\prime}\right) \frac{\left|\mathcal{M}_{\pi \pi}\right|^{2}}{16 E E_{1} E^{\prime} E_{1}^{\prime}} \times \\
& \quad \times f_{p}^{(0)} f_{p_{1}}^{(0)}\left(1+f_{p^{\prime}}^{(0)}\right)\left(1+f_{p_{1}^{\prime}}^{(0)}\right) P_{p}^{r}\left(\hat{p}^{i} \hat{p}^{j}-\frac{1}{3} \delta^{i j}\right) \times \\
& \quad \times\left[P_{p^{\prime}}^{s}\left(\hat{p}^{\prime} \hat{p}^{\prime j}-\frac{1}{3} \delta^{i j}\right)+P_{p_{1}^{\prime}}^{s}\left(\hat{p}_{1}^{\prime} \hat{p}_{1}^{\prime}{ }^{j}-\frac{1}{3} \delta^{i j}\right)\right. \\
& \left.\quad-P_{p}^{s}\left(\hat{p}^{i} \hat{p}^{j}-\frac{1}{3} \delta^{i j}\right)-P_{p_{1}}^{s}\left(\hat{p}_{1}^{i} \hat{p}_{1}^{j}-\frac{1}{3} \delta^{i j}\right)\right], \quad(\mathrm{A} 6)
\end{aligned}
$$

and

$$
C^{r}=-\frac{2}{3} \int \frac{\mathrm{d}^{3} p}{(2 \pi)^{3}} \frac{\vec{p}^{2}}{E T} f_{p}^{(0)}\left(1+f_{p}^{(0)}\right) P_{p}^{r} .
$$

The tensor $A^{r s}$ and the vector $C^{r}$ have to be calculated numerically. After that the coefficients $b_{r}$ are obtained by inverting eq. (A5).

For the numerical treatment, the expansion has to be truncated to a finite set of generalized Sonine polynomials. We have checked the convergence by studying the dependence of the resulting shear viscosity on the number of basis functions $N_{S}$. An example is given in fig. 12, where the $N_{S}$ dependence of $\eta$ is shown for $T=170 \mathrm{MeV}$. We find that it is sufficient to take into account the first three polynomials, a result which holds in the whole temperature range $T<T_{\text {Mott }}$.

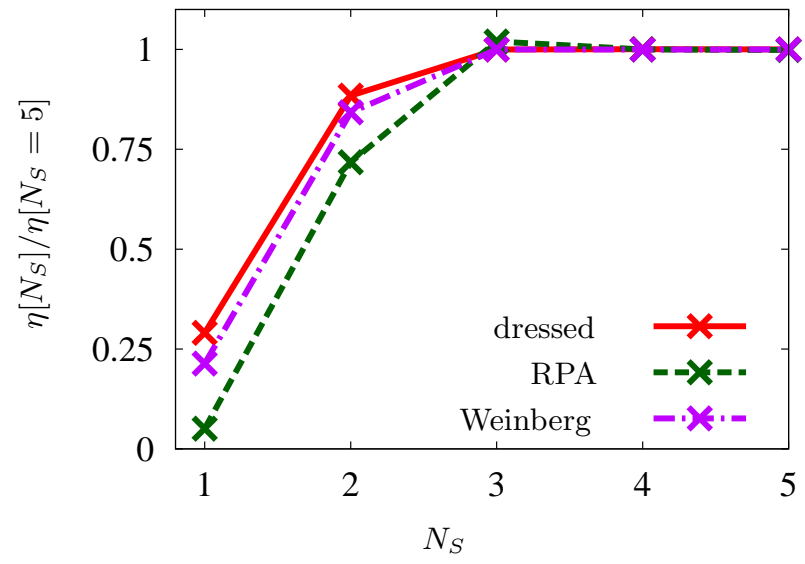

FIG. 12: Shear viscosity $\eta$ at $T=170 \mathrm{MeV}$ obtained with different numbers $N_{S}$ of generalized Sonine functions, normalized to the corresponding value for $N_{S}=5$. The points have been connected by lines to guide the eye. The different colors and line styles indicate different approximations for the scattering amplitude and are consistent with the choice in fig. 9 The result for the temperature dependent but momentum independent amplitude is identical to the result for the Weinberg amplitude, since the amplitudes differ only by a temperature dependent factor, which drops out by the normalization.
[1] P. Huovinen, P.F. Kolb, U.W. Heinz, P.V. Ruuskanen, S.A. Voloshin, Phys. Lett. B503, 58 (2001); hep-ph/0101136.

[2] D. Teaney, J. Lauret, E.V. Shuryak, Nucl. Phys. A698, 479 (2002); nucl-th/0104041.

[3] A. Adare et al. (PHENIX Collaboration), Phys. Rev. Lett. 98, 162301 (2007); nucl-ex/0608033.

[4] J. Adams et al. (STAR), Phys. Rev. C72, 014904 (2005), nucl-ex/0409033.

[5] M. Luzum, P. Romatschke, Phys. Rev. C 78, 034915 (2008), arXiv:0804.4015.

[6] M. Luzum, P. Romatschke, Phys. Rev. C 79, 039903 (2009); arXiv:0804.4015.

[7] H. Song, U. Heinz, J. Phys. G 36, 064033 (2009); arXiv:0812.4274.

[8] H. Niemi, G.S. Denicol, P. Huovinen, E. Molnar, D.H. Rischke (2011); arXiv:1101.2442.

[9] M. Prakash, M. Prakash, R. Venugopalan, G. Welke,
Phys. Rept. 227, 321 (1993).

[10] A. Dobado, J.R. Peláez, Phys. Rev. D 47, 4883 (1993); hep-ph/9301276.

[11] A. Dobado, J.R. Peláez, Phys. Rev. D 59, 034004 (1998); hep-ph/9806416.

[12] A. Dobado, S.N. Santalla, Phys. Rev. D65, 096011 (2002), hep-ph/0112299.

[13] A. Dobado, F.J. Llanes-Estrada, Phys. Rev. D 69, 116004 (2004); hep-ph/0309324.

[14] D. Fernández-Fraile, A. Gómez Nicola, Eur. Phys. J. A 31, 848 (2007); hep-ph/0610197.

[15] J. W. Chen and E. Nakano, Phys. Lett. B 647, 371 (2007); arXiv:hep-ph/0604138.

[16] J. W. Chen, Y. H. Li, Y. F. Liu and E. Nakano, Phys. Rev. D 76, 114011 (2007); arXiv:hep-ph/0703230.

[17] K. Itakura, O. Morimatsu, H. Otomo, Phys. Rev. D 77, 014014 (2008); arXiv:0711.1034.

[18] H. Lehmann, Physics Letters B 41, 529 (1972). 
[19] H. Pagels, Physics Reports 16, 219 (1975).

[20] S. Weinberg, Physica A 96, 327 (1979).

[21] J. Gasser, H. Leutwyler, Annals of Physics 158, 142 (1984).

[22] P. Gerber and H. Leutwyler, Nucl. Phys. B 321, 387 (1989).

[23] P. Gerber, H. Leutwyler and J. L. Goity, Phys. Lett. B 246, 513 (1990).

[24] Y. Nambu, G. Jona-Lasinio, Phys. Rev. 122, 345 (1961).

[25] Y. Nambu, G. Jona-Lasinio, Phys. Rev. 124, 246 (1961).

[26] U. Vogl and W. Weise, Prog. Part. Nucl. Phys. 27, 195 (1991).

[27] S.P. Klevansky, Rev. Mod. Phys. 64, 649 (1992).

[28] T. Hatsuda and T. Kunihiro, Phys. Rept. 247, 221 (1994); hep-ph/9401310.

[29] M. Buballa, Phys. Rep. 407, 205 (2005); hep-ph/0402234.

[30] A. Dobado, F.J. Llanes-Estrada, J.M. Torres-Rincon, Phys. Rev. D 80, 114015 (2009); arXiv:0907.5483.

[31] H.J. Schulze, J. Phys. G 21, 185 (1995).

[32] E. Quack, P. Zhuang, Y. Kalinovsky, S.P. Klevansky, J. Hüfner, Phys. Lett. B 348, 1 (1995); hep-ph/9410243.

[33] P.K. Kovtun, D.T. Son, A.O. Starinets, Phys. Rev. Lett. 94, 111601 (2005); hep-th/0405231.

[34] T. D. Cohen, Phys. Rev. Lett. 99, 021602 (2007); hep-th/0702136.

[35] S. Chapman, T.G. Cowling, The Mathematical Theory of Non-Uniform Gases (Cambridge University Press, 1970).

[36] S.R. de Groot, W.A. van Leeuwn, C.G. van Weert, Relativistic Kinetic Theory (North-Holland publishing com- pany, 1980).

[37] R.L. Liboff, Kinetic theory: classical, quantum, and relativistic descriptions (Springer, 2003).

[38] M. Oertel, M. Buballa and J. Wambach, Phys. Atom. Nucl. 64, 698 (2001); hep-ph/0008131.

[39] S. Gasiorowicz and D. A. Geffen, Rev. Mod. Phys. 41, 531 (1969).

[40] S. Weinberg, Phys. Rev. Lett. 17, 616 (1966).

[41] E. Quack and S. P. Klevansky, Phys. Rev. C 49, 3283 (1994).

[42] V. Dmitrasinovic, H. J. Schulze, R. Tegen and R. H. Lemmer, Annals Phys. 238, 332 (1995).

[43] M. Oertel, M. Buballa and J. Wambach, Phys. Lett. B 477, 77 (2000); hep-ph/9908475.

[44] M. Oertel, M. Buballa and J. Wambach, Nucl. Phys. A 676, 247 (2000); hep-ph/0001239.

[45] R. S. Plant and M. C. Birse, Nucl. Phys. A 703, 717 (2002); hep-ph/0007340.

[46] K. Goeke, M. M. Musakhanov and M. Siddikov, Phys. Rev. D 76, 076007 (2007); arXiv:0707.1997.

[47] J. Liao, V. Koch, Phys. Rev. C 81(1), 014902 (2010); arXiv: 0909.3105.

[48] A. Muronga, Phys. Rev. C 69, 044901 (2004); arXiv:nucl-th/0309056.

[49] M. I. Gorenstein, M. Hauer and O. N. Moroz, Phys. Rev. C 77, 024911 (2008); arXiv:0708.0137.

[50] J. Noronha-Hostler, J. Noronha and C. Greiner, Phys. Rev. Lett. 103, 172302 (2009); arXiv:0811.1571. 